\title{
Predicting Tumour Location by Simulating Large Deformations of the Breast Using a 3D Finite Element Model and Nonlinear Elasticity
}

\author{
Pras Pathmanathan ${ }^{1}$, David Gavaghan ${ }^{1}$, Jonathan Whiteley ${ }^{1}$, \\ Sir Michael Brady ${ }^{2}$, Martyn Nash ${ }^{3}$, Poul Nielsen ${ }^{3}$, and Vijay Rajagopal ${ }^{3}$ \\ 1 Oxford University Computing Laboratory, Parks Road, Oxford, UK \\ \{pras, gavaghan, jonathan.whiteley\}@comlab.ox.ac.uk \\ 2 Oxford University Department of Engineering Science, Parks Road, Oxford, UK \\ jmb@robots.ox.ac.uk \\ 3 Auckland University BioEngineering Institute, Auckland, New Zealand \\ \{martyn.nash,p.nielsen, v.rajagopal\}@auckland.ac.nz
}

\begin{abstract}
Two of the major imaging modalities used to detect and monitor breast cancer are (contrast enhanced) magnetic resonance (MR) imaging and mammography. Image fusion, including accurate registration between MR images and mammograms, or between CC and MLO mammograms, is increasingly key to patient management (for example in the multidisciplinary meeting), but registration is extremely difficult because the breast shape varies massively between the modalities, due both to the different postures of the patient for the two modalities and to the fact that the breast is forcibly compressed during mammography. In this paper, we develop a 3D, patient-specific, anatomically accurate, finite element model of the breast using MR images, which can be deformed in a physically realistic manner using nonlinear elasticity theory to simulate the breast during mammography.
\end{abstract}

\section{Introduction}

Breast cancer is one of the biggest killers of women in the Western world, killing 400,000 and affecting over a million people each year worldwide. As with all cancers, treatment can be lengthy, extremely unpleasant, and ultimately fruitless. Early diagnosis is the most effective way to improve the prognosis, and so screening programmes have been established in several western countries.

Currently, the imaging modalities used to image the breast are magnetic resonance (MR) imaging, mammography and ultrasound. Due to the highly deformable nature of the breast, and the difference in body position and external forces applied to the breast in the different imaging procedures, the shape of the breast varies massively between images of the different types, hindering attempts at image fusion for more effective diagnosis or predicting tumour location during surgery/biopsy. Various methods have been proposed to model breast deformation. Most are either based on linear elasticity theory, the theory used for 
small deformations, or to some extent on heuristics. In this paper, we describe the development of a finite element (FE) model of the breast based on the full nonlinear theory of elasticity (also known as finite elasticity). The FE mesh has a geometry determined from MR images of the subject, with an anatomical structure that is determined from segmented MR data. As such, we propose a patient-specific, not generic, model. Such a method models the deformation in a physically realistic manner, hence has a significantly higher likelihood of accurately simulating breast deformation than a linearised or heuristic method. Modelling large deformations with nonlinear elasticity has previously been considered computationally intractable; however, as we shall demonstrate, simulations on a moderately high-resolution mesh can be computed in a reasonable length of time on standard modern PC.

\section{Applications of a Physically Realistic Finite Element Model}

A robust, accurate deformable model of the breast would have many uses. Firstly, an accurate model could benefit image registration and fusion. For example, during mammography the patient's breast is forcibly compressed (typically $130 \mathrm{~N}$ ), so that fusing mammograms with MR data is extremely difficult. Also, in clinical practice mammograms are taken from different views, the most common being cranio-caudal (CC), (head-to-toe), and medio-lateral oblique (MLO), (shoulderto-opposite-hip). Combining the information provided by different mammogram views significantly enhances the detection of tumours, but the large deformation of the breast makes it hard for a radiologist to establish correspondences between these views, hence to reconstruct the tumour (or microcalcification cluster) in 3D. A deformable model of the breast (built from MR data) that can simulate both the CC compressed breast and the MLO compressed breast can be used to match the two types of mammogram. As well as inter-modality matching, the model can be used for temporal matching of images of the same modality.

MR images are generally carried out with the patient in the prone position. During surgery however, the patient will be supine, and a surgeon using MR data to predict the position of a tumour will have to resort to a degree of guesswork. A deformable model of the breast could be used to predict breast shape and tumour location during surgery, enabling minimally invasive surgery. Similarly, the model can be used as a guide during fine-needle or core biopsy, or even eventually to help automate the biopsy procedure.

Finally, the model we develop could be used as a visualisation tool, allowing a doctor to show the patient the position and size of the tumour in 3D, and as a teaching tool for radiologists. An extension of the model could also be used to simulate breast shape after reconstructive surgery.

Current methods used to model breast deformation are often not patientspecific, and/or they embody linear elastic or partially heuristic models of deformation. In [5] the breast edge is assumed to take the shape of quadratic curves, and compression is modelled by assuming that these curves map to new 
quadratic curves. In [4 the breast shape is obtained from patient data but the fibroglandular region is assumed to be cone shaped, and the tissues are assumed to be linearly elastic. In [1, a deformable MR based model of the breast is developed based on linear elasticity, where the large deformation is considered as a sequence of small linear deformations. However, the deformations the breast undergoes are extremely large and are not in the typical strain range assumed for small-strain linear elasticity (0-10\% strains). A physically-motivated nonlinear model is necessary to accurately model such deformations. Nonlinear models have, as far as we are aware, only been considered by Samani et al [8]. We aim to develop a fully nonlinear model with the ultimate goal of comparing nonlinear models of varying complexity with linear and simpler models to determine which factors are of greatest importance in breast deformation.

The modelling procedure is as follows: the FE geometry is built using the skin surface and pectoral muscle surface obtained from MR images. The MR images are (easily and accurately) segmented into regions of fat, fibroglandular and tumour tissue, which can be performed to sub-voxel precision. Using the segmented data, each element of the FE mesh is assigned a tissue type. The size of the elements in the mesh determine the potential accuracy of the deformation simulations. With a reasonably refined mesh accuracy to within $2-5 \mathrm{~mm}$ is possible, assuming the material laws of the tissues are known.

There are three distinct types of deformation which we have to simulate. The first is what we will call the forward problem, where body forces (e.g. gravity) are applied to an undeformed (unloaded) state to compute a deformed equilibrium state. This can be used to compute supine breast shape, for example. The second is the inverse problem (the backward problem): given a loaded deformed state, compute the unloaded undeformed state. This is necessary because the mesh built from MR data is initially in a gravity-loaded state. The third is modelling the compression of the breast during mammography. Here, the area of the skin in contact with the breast is unknown, and as such the boundary conditions are unknown. Such a problem is known as a contact problem.

\section{$3 \quad$ Nonlinear Elasticity}

In this section, we briefly formulate the problems which need to be solved. Let $\Omega_{0}$ be the undeformed state of the elastic body, let $\mathbf{X}$ be the position of a point in the undeformed state, let $\Omega$ be the deformed equilibrium state of the body (i.e. when it is loaded under gravity or external tractions), and let the unknown vector field $\mathbf{x} \equiv \mathbf{x}(\mathbf{X})$ be the position of that point in the deformed configuration. The deformation gradient is defined as the tensor $F_{i M}=\frac{\mathrm{d} x_{i}}{\mathrm{~d} X_{M}}$. The (Lagrangian) strain is defined as $E=\frac{1}{2}\left(F^{T} F-I\right)$. In nonlinear elasticity theory, a distinction is made between quantities defined in the undeformed coordinate system and those defined in the deformed coordinate system. Force balance equations can only initially be derived in the deformed equilibrium state, whereas the equations to be solved have to be written in terms of the known variables $\mathbf{X}$. It follows that the notion of stress can be defined in a number of ways, the most 
important being the Cauchy Stress, $\sigma_{i j}$, defined as the force acting on the deformed body measured per unit deformed area, and the Second Piola-Kirchoff Stress, $T_{M N}$, defined as the force acting on the undeformed body measured per unit undeformed area. $\sigma$ and $T$ are related by the expression $\operatorname{det}(F) \sigma=F T F^{T}$. In a gravity-loaded equilibrium deformed state all forces must balance, and it can be shown [6] that this leads to

$$
\frac{\partial \sigma_{i j}}{\partial x_{j}}+\rho g_{i}=0 \quad \text { in } \Omega,
$$

where $\rho$ is the density in the deformed body and $\mathbf{g}$ is gravitational acceleration. This equation has to be reformed in terms of the known variables, leading to the following equilibrium equation

$$
\frac{\partial}{\partial X_{M}}\left(T_{M N} \frac{\partial x_{i}}{\partial X_{N}}\right)+\rho_{0} g_{i}=0 \quad \text { in } \Omega_{0},
$$

where $\rho_{0}$ is the density in the undeformed body.

To relate stress to strain, we need the material dependent constitutive relation between them. Linear stress-strain relationships are often assumed in linear elasticity (small-strain elasticity); however, biological tissues have been shown to exhibit nonlinear stress-strain laws [2] and this is certainly the case for the range of substantial strains involved in breast deformation, so we consider the full nonlinear laws for our model. To do so, we assume there exists a strain energy function $W \equiv W\left(E_{M N}\right)$ for each tissue which satisfies

$$
\frac{\partial W}{\partial E_{M N}}=T_{M N} .
$$

Note that in this equation we ignore viscoelastic effects, which we assume to be negligible on the long-timescale problems we are considering. The constitutive law for each tissue can only be determined experimentally. The relative lack of experimentally-determined tissue material laws is perhaps the major factor inhibiting the use of nonlinear elasticity in modelling large biological deformations.

As discussed in Section 2, there are three types of problems which need to be solved:

The Forward Problem: Here the undeformed breast shape is given and the breast shape under gravity (say) is required, a standard elasticity problem. For this, we need to solve (2), together with tissue material laws of the form (31), for $\mathbf{x}$, subject to zero displacement boundary conditions on the part of the mesh corresponding to the pectoral muscle and zero surface pressure boundary conditions on the skin surface.

The Backward Problem: A mesh built from MR data will typically be for the breast under the influence of gravity. We initially need to calculate the unloaded state (the shape the breast would take in the absence of gravity) before any other deformation calculations can be computed. This involves solving an inverse finite elasticity problem: we know the deformed position $\mathbf{x}$ and wish to 
compute $\mathbf{X}$. This can be accomplished by solving (11), with the material law (3), but writing the strain as $E=\frac{1}{2}\left(\left(G^{-1}\right)^{T}\left(G^{-1}\right)-I\right)$, where $G=\frac{\partial \mathbf{X}}{\partial \mathbf{x}}=F^{-1}$, so that $E$ is a function of $\mathbf{x}$, and using the relationship between $\sigma$ and $T$ to obtain $\sigma$ from the material law.

The Contact Problem: The problem of modelling breast compression has to be considered separately from the other two types of problem. In this case, we do not know which part of the breast will be in contact with the compression plates, and therefore cannot just specify displacement boundary conditions. To derive the contact problem formulation, it is necessary to reformulate (2) as an energy minimisation problem:

$$
\min \int_{\Omega_{0}} W\left(E_{M N}\right)-\rho_{0} \mathbf{g} \cdot \mathbf{x} \mathrm{d} V_{0} \quad \text { over } \mathbf{x} \text { satisfying b.c.s, }
$$

where the boundary conditions (b.c.s) are that the displacement is zero on the pectoral muscle. The (frictionless) contact problem can now be stated as:

$$
\min \int_{\Omega_{0}} W\left(E_{M N}\right)-\rho_{0} \mathbf{g} \cdot \mathbf{x} \mathrm{d} V_{0} \quad \text { over } \mathbf{x} \text { satisfying b.c.s and constraints, }
$$

where the constraints are that no point penetrates either compression plate. One method of solving such a problem is to add a penalty function to the energy and minimise over unconstrained $\mathbf{x}$ :

$\min \int_{\Omega_{0}} W-\rho_{0} \mathbf{g} \cdot \mathbf{x} \mathrm{d} V_{0}+\int_{\Gamma} \frac{P}{2}\left([-g(\mathbf{x}(\mathbf{X}))]_{+}\right)^{2} \mathrm{~d} S_{0} \quad$ over $\mathbf{x}$ satisfying b.c.s,

where $\Gamma$ is the skin surface and $g(\mathbf{x})$ is the signed normal distance of a point $\mathbf{x}$ to the nearest plate, and $[z]_{+}$is 0 if $z \leq 0$ and $z$ if $z>0 .[-g(\mathbf{x})]_{+}$is therefore the penetration of a point into the plates, which we wish to be zero. The penalty function penalises violation of the constraint, with greater values of the penalty parameter $P$ leading to greater penalisation.

A well-known drawback of penalty functions is that $P$ needs to be large in order to assure accurate results, but this can lead to badly conditioned matrices when the problem is solved with the finite element method. The Augmented Lagrangian Method, which we have used, is an extension of the penalty function method which deals with this problem. For details see [3], but the basic idea is that the penalty function is replaced with

$$
\int_{\Gamma} \frac{1}{2 P}\left([-\lambda(\mathbf{x})-P g(\mathbf{x})]_{+}\right)^{2} \mathrm{~d} S_{0}
$$

where $\lambda$ is an estimate of the Lagrange multipliers associated with the constraint (which are also the compressive forces applied by the plates) that is updated iteratively and converges to the true Lagrange multipliers. With this method $P$ need not be very large to guarantee accurate results. 


\section{Modelling and Results}

A finite element model has been built from MR data of a standing patient. A mesh hexahedral elements was created and adapted to the breast geometry, by fitting the front surface of the mesh to the skin surface and the back surface of the mesh to the pectoral muscle surface. Hexahedral elements are preferred to tetrahedral elements since they have been shown to have better convergence properties [8]. The next stage would be to assign a tissue to each element based on segmented MR data; however with these preliminary results each element has just been considered to be fibroglandular tissue. The final mesh has 5625 nodes and 4608 elements, with trilinear basis functions used to interpolate position, and is shown in Figure 1 .

We have assumed the breast tissues are incompressible, since breast tissues are primarily comprised of water. We have currently also made the incorrect assumption that the tissues are isotropic. Breast tissues are likely to be anisotropic, since the connective structures known as Cooper's Ligaments are oriented in the muscle-to-skin direction. In future work, we will investigate the effect of modelling tissues as transverse isotropic or fully anisotropic. If a material is isotropic, the constitutive law for the material simplifies somewhat, in this case the strain energy function $W$ satisfies $W \equiv W\left(I_{1}, I_{2}, I_{3}\right)$, where $I_{1}, I_{2}$ and $I_{3}$ are the principal invariant] of $F^{T} F$ [ . We have assumed the exponential constitutive law

$$
W\left(I_{1}, I_{2}, I_{3}\right)=a\left(e^{b\left(I_{1}-3\right)}-1\right)-\frac{p}{2}\left(I_{3}-1\right),
$$

where $a$ and $b$ are material constants and $p$ is the Lagrange multiplier associated with the incompressibility constraint which can be interpreted as internal pressure. We have estimated $a$ and $b$ using experimental data in [10].

The equations discussed in Section 3 are solved on the mesh using the Galerkin finite element method [7. For incompressible elastic deformations, the internal pressure $p$ has to be computed together with the displacements. Piecewise constant basis functions are used to interpolate the pressure, as the pressure must be interpolated by lower order basis functions than the position [7]. Since the equations are highly nonlinear, Newton's method has been used.

The results of two simulations on this mesh are shown in Figures 10 and 2 Figure 1 displays the undeformed breast shape which is the result of solving the backward problem on the mesh built from the MR data, together with a supine simulation. Figure 2 is an example of a simulation of CC mammographic compression. The simulations of the supine and compressed breast shape take less than 1.5 hours on a $2 \mathrm{GHz}$ Linux PC. Due to badly-conditioned matrices inherent in the backward problem, computing the undeformed state currently takes significantly longer, up to 24 hours on a $2 \mathrm{GHz}$ Linux PC. Preconditioning the backward problem matrices or finding methods of improving Newton convergence to reduce the time to solve the backward problem is an open problem. In later work will verify these simulations using both phantom models and

\footnotetext{
${ }^{1}$ The principal invariants of a matrix $C$ are $I_{1}=\operatorname{tr}(C), I_{2}=\frac{1}{2}\left((\operatorname{tr}(C))^{2}-\operatorname{tr}\left(C^{2}\right)\right)$ and $I_{3}=\operatorname{det}(C)$.
} 

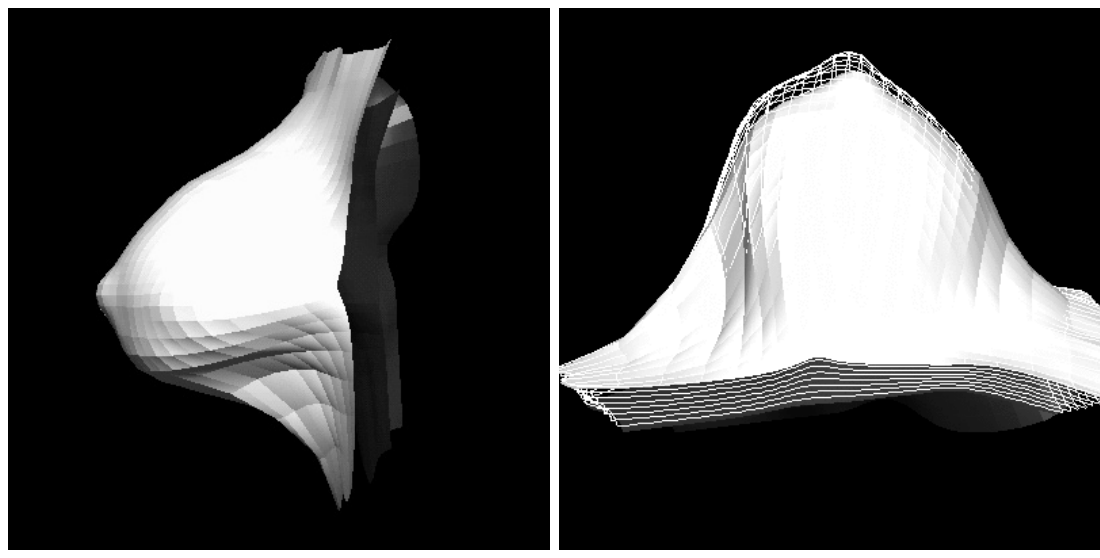

Fig. 1. Left: mesh of the breast built from MR images of a standing patient, right: the undeformed (gravity-loading removed) breast shape (wireframe) and supine patient breast shape (surface)

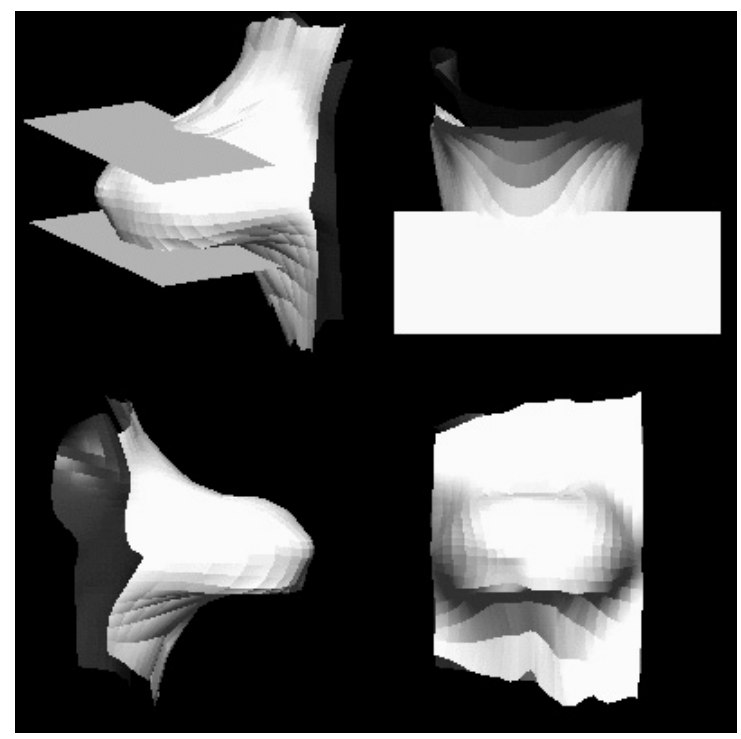

Fig. 2. Various views of the breast under simulated CC mammographic compression

patient data. The latter will involve reproducing supine or compressed breast shape and comparing the surface deformation with photographic results, and simulating the breast shape with a new direction of gravity and comparing with MR images. 


\section{Conclusions}

In this paper we have demonstrated that using the full nonlinear formulation to model the large deformations the breast undergoes is not a computationally intractable problem, and have used a nonlinear patient-specific finite element model to predict surgery breast shape and breast shape under mammographic compression. This method can ultimately be used to perform image matching and predict tumour location for surgery or biopsy. Current work is aimed at validating the simulations. We have described the three types of deformation problem which have to be tackled, and outlined procedures for solving them. In future work we will perform simulations with heterogeneity obtained from segmented MR images, investigate the effect of material parameters on the deformations, and investigate whether the tissues have to be modelled as transversely isotropic or anisotropic. We will also model mammographic compression as a frictional contact problem. Verification using both phantom and patient data studies will be performed.

\section{References}

1. F.S. Azar, D.N. Metaxas, M.D. Schnall. A Deformable Finite Element Model of the Breast for Predicting Mechanical Deformations under External Perturbations, Acad. Rad. Oct 2001.

2. Y.C. Fung. Biomechanics: Mechanical Properties of Living Tissues, SpringerVerlag, 1993.

3. G. Kloosterman. Contact Methods in Finite Element Simulations (PhD Thesis), University of Twente, 2001

4. A.M Galea, R.D. Howe. Mammography Registered Tactile Imaging, Surgery Simulation and Soft Tissue Modeling, International Symp. IS4TM 2003 Proc, Springer

5. Y. Kita, R.P Highnam, J.M. Brady. Correspondence between Different View Breast X-Rays Using Curved Epipolar Lines. Computer Vision \& Image Understanding 83.

6. M. Nash. Mechanics and Material Properties of the Heart using an Anatomically Accurate Mathematical Model (PhD Thesis), University of Auckland, 1998

7. J.N. Reddy. An Introduction to the Finite Element Method, McGraw-Hill 1993

8. A. Samani, J. Bishop, E. Ramsay, D.B. Plewes. A 3-D Contact Problem Finite Element Model for Breast Shape Deformation Derived From MRI Data. Proc. of ASB, Annual Conference, 1999

9. A. J. M. Spencer. Continuum Mechanics, Longman 1980

10. P. Wellman, R.H. Howe, E. Dalton, and K.A. Kern. Breast Tissue Stiffness in Compression is Correlated to Histological Diagnosis. Technical Report, Harvard Biorobotics Laboratory Technical Report, 1999 